\title{
Macrophage: SHIP of Immunity
}

\author{
Charles D. Mills ${ }^{1}{ }^{*}$, Anita C. Thomas ${ }^{2}$, Laurel L. Lenz ${ }^{3}$ and Markus Munder ${ }^{4}$ \\ BioMedical Consultants, Marine on St. Croix, MN, USA \\ ${ }^{2}$ Bristol Heart Institute and Bristol CardioVascular, University of Bristol, Bristol, UK \\ ${ }^{3}$ Immunology and Microbiology Department, University of Colorado School of Medicine, Aurora, CO, USA \\ ${ }^{4}$ Third Department of Medicine, University Medical Center, Johannes Gutenberg University, Mainz, Germany \\ *Correspondence: mills002@umn.edu \\ Edited by: \\ Claudia Kemper, King's College London, UK \\ Reviewed by: \\ Geanncarlo Lugo-Villarino, Centre National de la Recherche Scientifique, France
}

Keywords: macrophage, M1, M2, nitric oxide, iNOS, arginase, wound, cancer

Immunology. Why does it exist? Two words. Cure disease. People get diseases. "Test tubes" do not. People fund immunologists for solutions to their health problems. But, immunologists often study leukocytes in test tubes - the laboratory - away from diseases. Why? Because much can be learned from analyzing cellular biochemistry and behaviors in vitro that cannot be ascertained when leukocytes are in animals. At the same time, isolated leukocyte reactions often do not reflect how the immune system operates as a unit. So, it is critical to verify in vitro observations in vivo. Among leukocytes, macrophages are the central initiating and directing element in immune systems, and serve this role through four basic "SHIP" functions in vivo: Sample; Heal; Inhibit; and Present (antigen) (1-4). The polar-opposite functions of Heal (M2type) and Inhibit (M1-type) can have profoundly different effects on host survival, and require unique and major changes in macrophage metabolism and physiology. In turn, macrophage populations are necessarily heterogeneous as they adapt to protect hosts in different ways: they exhibit "plasticity." Some have focused on measuring ever-expanding lists of cell surface or various other "markers" (mostly in vitro) to try and sub-type macrophages. But, the "heterogeneity" created by such studies can be "illusory" because there are many more markers than there are functions (e.g., M1/inhibit and M2/heal). Thus, it is important to focus on classifying macrophages by functions, such as SHIP, to navigate through a "sea of plasticity." And, thereby realize the enormous potential of macrophages/innate immunity for improving health.

\section{BASIC MACROPHAGE FUNCTIONS IN VIVO}

The earliest in vivo SHIP function observed in macrophages was their ability to "sample" by ingesting items in their surroundings $(5,6)$. Through sampling, macrophages routinely receive "self" signals that instruct them to repair or replace lost or effete cells and intercellular matrices. The heal-type function of macrophages is now called M2 [(7), reviewed in Ref. (2)]. Following infection (or trauma), M2/healtype macrophages can rapidly switch to become M1/inhibit-type, to promote host defense (1). M2/heal responses are mediated by ornithine, and other growthpromoting molecules $(8,9)$. M1/inhibit is mediated by nitric oxide (NO) and other molecules that promote cellular killing activity $(10,11)$. Fascinatingly, both ornithine and NO arise from one amino acid: arginine (12).

The biochemical basis for the M2/heal function of macrophages was discovered before the M1/inhibit function $(8,12)$. As illustrated in Figure 1A (top), in sterile wounds, macrophages produce ornithine (a precursor of polyamines and collagen for repair) as healing proceeds (Green - M2 dominance) (13). Around the same time, it was observed that macrophages in growing tumors were also the M2/ornithineproducing type (Figure 1A middle). This latter finding provided a biological explanation for the association of intratumor macrophages with tumor growth (14). M2/heal-type macrophages have since been shown to also dominate in human tumors, and are associated with poor survival (15-18).

The biochemical basis of how M1/ inhibit-type macrophages kill pathogens (or, abnormal "self") also came from the study of wounds and cancer described above, as well as other studies [(1926), reviewed in Ref. $(3,12)]$. As mentioned, macrophages have a unique ability to switch from making the heal molecule, ornithine, in vivo to making the killer molecule, NO (1). Such a switch is shown in Figure 1A (top). For a brief period following wounding, a high concentration of NO is present (M1 activity, Red), which can protect the wound if infectious agents have been introduced (13). It is not clear exactly what stimuli cause this injury-induced NO production, though neutrophils are also involved (27, 28 ). If the wound is sterile, macrophage NO production stops, neutrophil emigration ends, and wound macrophages make ornithine (M2 activity) as mentioned. Another example of the key protective function of macrophages making $\mathrm{NO}$ is shown in Figure 1A (bottom). It can be seen that if a host is vaccinated against the tumor shown in Figure 1A (middle), implantation of the tumor causes intratumor macrophages to make a large quantity of NO that helps cause tumor rejection (12). Macrophage NO is also an important defense against a variety of infectious diseases (29). That M2/ornithine or M1/NO are important effector molecules are supported by studies showing that interference with these activities in vivo alters healing or host protection [reviewed in Ref. (12)]. Conversely, overexpression of $\mathrm{M} 1 /$ inhibit responses is associated with conditions such as atherosclerosis and arthritis, while M2-type contribute to chronic infections, promotion of tumor growth, and allergies (3, 29). Together, these results demonstrated 


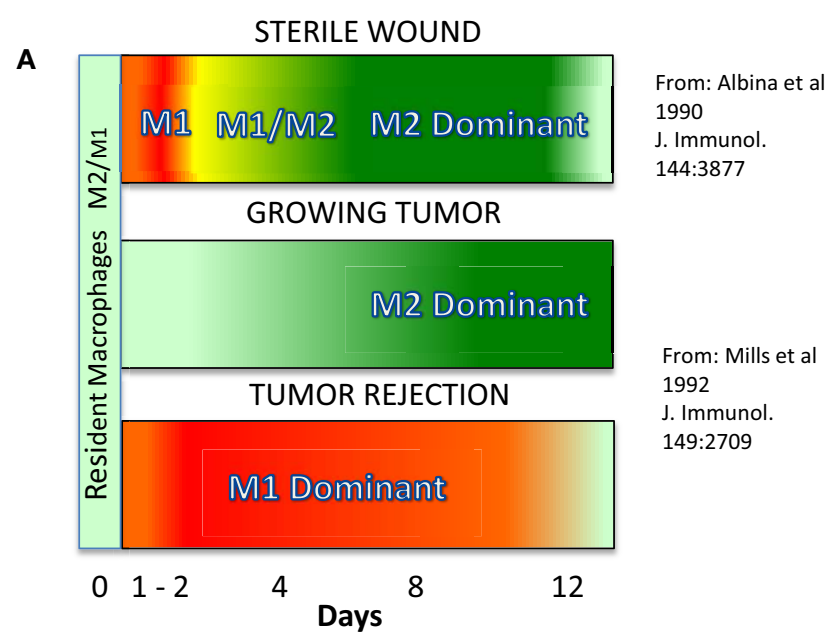

B

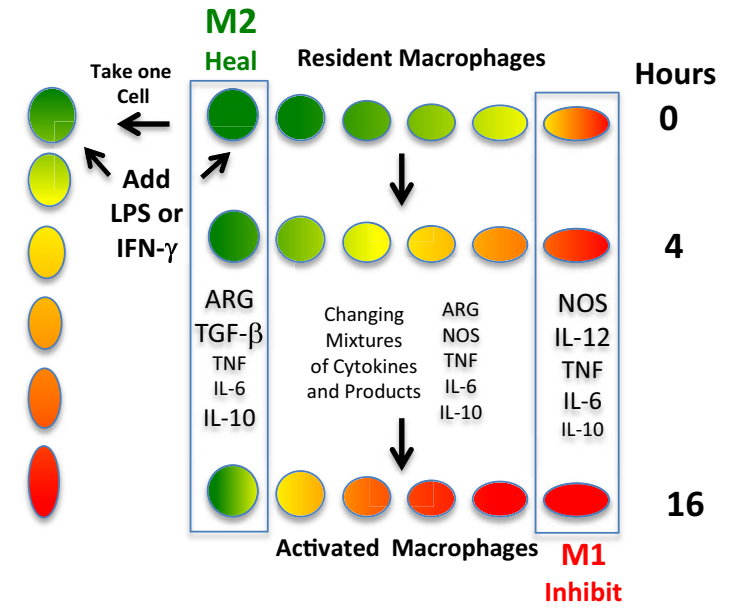

FIGURE 1 | (A). Predominance of M1 or M2-dominant macrophage responses in vivo in different types of inflammation. Top: a wound results in a strong, but brief, shift from local macrophages making primarily ornithine to making NO. Wound macrophages then become M2-dominant to aid in wound healing. Middle: a growing tumor elicits macrophages, and macrophage production of $\mathrm{NO}$ is suppressed and ornithine production predominates. Bottom: if a host is vaccinated against a tumor, implantation of the tumor results in T cell-dependent M1 dominance and the tumor is rejected. (B) M2 to M1-type macrophage conversion and the illusion of "subsets." Top: resident macrophages primarily produce ornithine via arginase (Arg). Middle: upon stimulation, macrophage production of iNOS/NO increases. And the production of other cytokines, etc., changes during this time. Bottom: after $16 \mathrm{~h}$, macrophage NO production increases further and ornithine production declines.

two core functions that result from macrophages sampling their environment, and that affect health in very important, and opposite, ways: the M1/inhibit response and the $\mathrm{M} 2 /$ heal response.

Inhibit-type macrophages and healtype macrophages were specifically renamed M1 and M2 because these macrophage responses [or dendritic cells ${ }^{1}$
(30-32)] were also found to stimulate $\mathrm{T}$ cells to make Th1-type (cellular-based), or Th2-type (antibody-based) cytokines (7), respectively. This fourth SHIP function of present (antigen) is only expressed in vertebrates (1). Although T cells can produce molecules that "activate" or "alternatively activate" macrophages $(33,34)$, macrophages evolved first and respond first. They directly sense Pathogen or Damage - Associated Molecular Patterns (PAMP or DAMP) that can initiate M1 or M2-type responses (35-39). Subsequently, macrophages can stimulate T cells (that cannot recognize antigens directly), and thereby further amplify M1 or M2 responses. This "secondary" type of T celldriven response (macrophage "activation") was discovered by Mackaness and colleagues using mice preimmunized to Listeria and other pathogens (40). It was not known at this time that macrophages were actually responsible for initially activating $\mathrm{T}$ cells $(41,42)$. The recent revelation about the central role of macrophages in immune responses caused a sea change in understanding how immune responses occur and are regulated in vivo $(1,7)$.

\section{BIOCHEMICAL AND PHYSIOLOGIC HOST ELEMENTS THAT INFLUENCE HOW MACROPHAGES PROTECT HOSTS}

Macrophage SHIP functions (sample, heal, inhibit, and present) are regulated by integration of a variety of endogenous (e.g., host-derived and resident microbiota) and exogenous signals $(1,43)$. For example, in the absence of infection or injury, TGF- $\beta$ helps maintain macrophages in the routine M2/heal mode (7). Other host-derived molecules, such as oxidized LDL, can stimulate M1-type responses that contribute to atherosclerosis $(4,44)$. Following infection or injury, certain PAMPs and DAMPs stimulate macrophages to switch from M2/heal to $\mathrm{M} 1 /$ inhibit mode (35-37). IFN- $\gamma$ was shown to be the primary $\mathrm{T}$ cell product that further amplifies M1/inhibit activity (45). Later, macrophage IL-12 was found to be a key cytokine (along with increased Class II MHC expression) that stimulates IFN- $\gamma$ production by $T$ cells $(41,42,46)$. Macrophages have also been reported to secrete IFN- $\gamma$ upon stimulation via IL-12 and IL-18 (47) or via CD40 (48), which might further enhance M1 polarization through auto- or paracrine activity. Not all pathogens stimulate macrophages to switch from M2/heal to M1/inhibit, and some seem to suppress such a switch. In this circumstance, M2-type macrophages can stimulate $\mathrm{T}$ cells to make very different cytokines (such as IL-4, IL-13, and

${ }^{1}$ Both macrophages and dendritic cells can direct the type of $\mathrm{T}$ cell response. So, the word "macrophage" will be used here to refer to both. Readers are directed elsewhere for discussions of macrophages and dendritic cells. 
TGF- $\beta$ ) that cause B cells to become antibody-producing plasma cells $(7,29)$. These same cytokines also inhibit the M2 to M1 switch, and thus can amplify M2/heal activity (1). Of course, because there are many different pathogens invading different locales of hosts, there are always mixtures of M1/Th1 or M2/Th2type responses as disease regression or progression occurs. In this connection, it is now known that tissue macrophages can arise from local renewal or from the blood $(1,4)$. The ontogeny of M1 and M2type macrophages is not yet clear, and is beyond the scope of this article. Recent advances in metabolomics, defining resident microbiota, other areas, are opening up new horizons for understanding the myriad signals that regulate "immunity" (43). Though more is to be known, the aforementioned results have established important biochemical and physiologic elements that influence how macrophages serve to protect (or fail to protect) against infectious or other threats to host homeostasis.

\section{IN VITRO VERSUS IN VIVO MACROPHAGE CONUNDRUM}

In addition to the basic macrophage functions necessary for life (such as SHIP), some investigators (primarily working in vitro) have employed ever-expanding lists of "markers" for macrophage "activation." These include: cell surface antigens; expressed gene products; and other factors, and have created the notion that there are many different "varieties" of macrophages such as "M2 a, b, c," "regulatory," and "alternatively activated" macrophages (49-53). Unlike classifying macrophages by functions (e.g., M1/inhibit or M2/heal), the use of markers has created subsets without clear functional roles in vivo. Likewise, defining macrophage populations based on cytokine production patterns has caveats that are often overlooked. For example, macrophage cytokines such as IL-6 are "inflammatory," yet they can be found in almost any site where macrophages are present (1). Indeed, the very presence of macrophages is inflammatory that raises questions about what "anti-inflammatory" macrophages are (47-49). Efforts to define macrophage "subsets" based on which cytokine (or agonist) has been used to stimulate them in vitro (such as IL-4 or IFN- $\gamma$ ) also leads to confusion since macrophages do not encounter isolated cytokines in vivo. Rather, they are constantly receiving hundreds of signals, the integration of which ultimately defines a cell's behavior. Furthermore, because a selected cytokine can elicit a given macrophage reaction in vitro does not mean it has the same effect in vivo. For example, adding IL4 to macrophages in vitro does increase M2-type activity (50). And IL-4 from T cells or innate cells can upregulate M2type antibody responses $(7,29)$ : what has been has been termed "alternative activation"). However, it is hard to ascribe M2-type responses in circumstances such as sterile wounds or tumors to "alternative activation" because little or no IL-4 is present $(54,55)$. Using $\mathrm{T}$ cell-derived cytokines to stimulate macrophages in vitro has also propagated the long-held notion that $\mathrm{T}$ cells are necessary to "activate" macrophages $(23,24)$. This perception runs counter to the observations that macrophages initiate and direct innate or adaptive responses (1). Another potential artifact of in vitro cultures is that macrophages can exhaust critical media components, and thus behave in ways (including dying) that are not observed in vivo where nutrients/other products are replenished (24).

Finally, the source of the "macrophages" being studied in vitro varies and has created confusion. Specifically, people studying humans have primarily used monocytes from blood because of convenience. And doing so has caused some to conclude there are major species differences in "macrophages," including that humans seem less able (or unable) to produce iNOS/NO or arginase/ornithine $(3,56,57)$. However, comparing monocyte-derived macrophages to tissue macrophages is an apples and oranges-type comparison. When human tissue macrophages have been examined, they do not appear fundamentally different from those of other vertebrate species (58).

Thus, a variety of pitfalls can make it difficult to translate results from in vitro cultures to understanding how macrophages function in vivo. In turn, rather than relying on "markers" or selected culture stimuli to try and define different macrophage "activation" states (59), it seems prudent to focus on characterizing macrophages by their known in vivo functions, such as SHIP (1).

\section{SHIP FUNCTIONS TO NAVIGATE A SEA OF PLASTICITY}

Macrophage SHIP functions are associated with major differences in their metabolism and physiology (1). And hence, at the population level, macrophages must display considerable heterogeneity. "Plasticity" usefully describes the unique adaptability of macrophages as they change from, for example, producing a growthpromoting molecule (ornithine) to producing a growth-inhibiting molecule (NO) $(12,60)$. However, for some the concept of plasticity has morphed into a notion that macrophages are a fluid cell type that are always only changing (47-51). Like they say, "change is good" (humor intended). But, like changing clothes, it is not the changing that matters: it is the result. Perhaps, the clothes help one get a job, or, get a date, etc. And so it is with macrophages. As macrophages make major switches in their metabolism, they are "changing." But, the changes in functional properties of macrophages can create illusory heterogeneity as illustrated in Figure 1B. Specifically, if a population of resting/resident macrophages (or a single macrophage, left) receives appropriate signals (e.g., LPS and/or IFN- $\gamma$ ) and commits to switching from $\mathrm{M} 2 /$ heal to $\mathrm{M} 1$ /inhibit dominant activity, it takes the cell(s) several hours to accomplish this major change in metabolism. In turn, at any given time there will be a variety of different macrophages expressing different M2 and (increasingly in this example) M1-type activity. In turn, if one examines macrophages (or a single macrophage) at any given time there will be intermediate phenotypes in terms of marker or cytokine expression. Also often overlooked is that M1-type macrophages produce non-specific killer molecules (like NO) that inhibit or kill macrophages too (24). In turn, analysis of whole populations can create the additional illusion that M1-type have converted back to M2type, when actually, they are dead/missing (1) In turn, examining macrophage populations (particularly in vitro) can create impressions of reversible plasticity or heterogeneity, but which are not based on what functions the macrophages have (e.g., M1/inhibit or M2/heal) (49). Thus, 
heterogeneity (or plasticity) is a means to an end. The "end" immunologists should strive for is identifying macrophages by their health-impacting functions (1).

\section{SUMMARY}

Immunology has and will continue to cure important diseases. And, the ability to culture macrophages in vitro, the expanding power of "transcriptome" analysis to examine thousands of genes, the capability of analyzing single macrophages, and other new technologies are providing necessary new information about the cellular biochemistry and physiology of leukocytes. But, as demonstrated here with macrophages, overemphasis on ambiguous "markers," or analyzing whole populations of macrophages that are changing their functions, can create an illusion - a "sea of plasticity." Therefore, to navigate this sea, it is critical to focus on SHIP functions (e.g., sample, heal, inhibit, and present) that importantly affect health. Doing so will help unleash the tremendous potential for usefully modulating innate immunity/macrophages against a variety of conditions ranging from cancer to atherosclerosis. To cure disease.

\section{ACKNOWLEDGMENTS}

We appreciate the excellent and unselfish input we have received from researchers in forming this paper. Namely, because we are all on the "same team": the goal being better health.

\section{REFERENCES}

1. Mills CD, Ley K. M1 and M2 macrophages: the chicken and the egg of immunity. J Innate Immun (2014) 6:716-26. doi:10.1159/000364945

2. Wynn TA, Chawla A, Pollard JW. Macrophage biology in development, homeostasis and disease. Nature (2013) 496:445-55. doi:10.1038/ nature 12034

3. Mills CD. M1 and M2 macrophages: oracles of health and disease. Crit Rev Immunol (2012) 32:463-88. doi:10.1615/CritRevImmunol. v32.i6.10

4. Geissmann F, Manz MG, Jung S, Sieweke MH, Merad M, Ley K. Development of monocytes, macrophages, and dendritic cells. Science (2010) 327:656-61. doi:10.1126/science.1178331

5. Cooper EL. eCAM: Darwin and Metchnikoff. Evid Based Complement Alternat Med (2009) 6:421-2. doi:10.1093/ecam/nep194

6. Dzik JM. The ancestry and cumulative evolution of immune reactions. Acta Biochim Pol (2010) 57:443-66.

7. Mills CD, Kincaid K, Alt JM, Heilman MJ, Hill AM. M-1/M-2 macrophages and the Th1/Th2 paradigm. J Immunol (2000) 164:6166-73. doi:10. 4049/jimmunol.164.12.6166

8. Morris SM. Recent advances in arginine metabolism: roles and regulation of the arginases. $\mathrm{Br}$ J Pharmacol (2009) 6:922-30. doi:10.1111/j.14765381.2009.00278.x

9. Munder M. Arginase: an emerging key player in the mammalian immune system. Br J Pharmacol (2009) 158:638-51. doi:10.1111/j.1476-5381.2009. 00291.x

10. Hibbs JB, Taintor RR, Vavrin Z, Rachlin EM. Nitric oxide: a cytotoxic activated macrophage effector molecule. Biochem Biophys Res Commun (1988) 157:87-94. doi:10.1016/S0006-291X(88) 80015-9

11. Nathan CF. Nitric oxide as a secretory product of mammalian cells. FASEB J (1992) 12:3051-64.

12. Mills CD. Macrophage arginine metabolism to ornithine/urea or nitric oxide/citrulline: a life or death issue. Crit Rev Immunol (2001) 21:399-426. doi:10.1615/CritRevImmunol.v21.i5.10

13. Albina JE, Mills CD, Henry WL Jr, Caldwell MD. Temporal expression of different pathways of 1arginine metabolism in healing wounds. JImmunol (1990) 144:3877-80.

14. Mills CD, Shearer J, Evans R, Caldwell MD. Macrophage arginine metabolism and the inhibition or stimulation of cancer. J Immunol (1992) 149:2709-14.

15. Komohara Y, Jinushi M, Takeya M. Clinical significance of macrophage heterogeneity in human malignant tumors. Cancer Sci (2014) 105:1-8. doi: $10.1111 /$ cas. 12314

16. Noy R, Pollard JW. Tumor-associated macrophages: from mechanisms to therapy. Immunity (2014) 41:49-61. doi:10.1016/j.immuni. 2014.06.010

17. Tang X, Mo C, Wang Y, Wei D, Xiao H. Anti-tumour strategies aiming to target tumour-associated macrophages. Immunology (2012) 138:93-104. doi:10.1111/imm.12023

18. Weigert A, Brune B. Nitric oxide, apoptosis and macrophage polarization during tumor progression. Nitric Oxide (2008) 2:95-102. doi:10.1016/j. niox.2008.04.021

19. Nathan C. Role of iNOS in human host defense. Science (2006) 312:1874-5. doi:10.1126/science. $312.5782 .1874 \mathrm{~b}$

20. Hibbs JB. Infection and nitric oxide. J Infect Dis (2002) 185:S9-17. doi:10.1086/338005

21. Vogel SN, Johnson D, Perera PY, Medvedev A, Lariviere L, Qureshi ST, et al. Cutting edge: functional characterization of the effect of the $\mathrm{C} 3 \mathrm{H} / \mathrm{HeJ}$ defect in mice that lack an Lpsn gene: in vivo evidence for a dominant negative mutation. J Immunol (1999) 162:5666-70.

22. Reiss CS, Komatsu T. Does nitric oxide play a critical role in viral infections? J Virol (1998) 78:4547-51.

23. Stevens RB, Ansite JD, Mills CD, Lokeh A, Rossini TJ, Saxena M, et al. Nitric oxide mediates early dysfunction of rat and mouse islets after transplantation. Transplantation (1996) 12:1740-9. doi:10. 1097/00007890-199606270-00014

24. Albina JE, Caldwell MS, Henry WL, Mills CD. Regulation of macrophage functions by L-arginine. $J$ Exp Med (1989) 169:1021-9. doi:10.1084/jem.169. 3.1021
25. Nathan CF. Secretory products of macrophages. J Clin Invest (1987) 79:319-26. doi:10.1172/ JCI112815

26. Hibbs JB, Vavrin Z, Taintor RR. L-arginine is required for expression of the activated macrophage effector mechanism causing selective metabolic inhibition in target cells. J Immunol (1987) 138:550-65.

27. Li Y, Karlin A, Loike JD, Silverstein SC. Determination of the critical concentration of neutrophils required to block bacterial growth in tissues. J Exp Med (2004) 200:613-22. doi:10.1084/ jem.20040725

28. Filipin L, Moreira AJ, Marroni NP, Xavier RM. Nitric oxide and repair of skeletal muscle injury. Nitric Oxide (2009) 3:157-63. doi:10.1016/j.niox. 2009.08.002

29. Murray PJ, Wynn TA. Protective and pathogenic functions of macrophage subsets. Nat Rev Immunol (2011) 11:723-37. doi:10.1038/ nri3073

30. Geissmann F, Gordon S, Hume DA, Mowat DA, Randolph GJ. Unravelling mononuclear phagocyte heterogeneity. Nat Rev Immunol (2010) 10:453-60. doi: $10.1038 /$ nri2784

31. Hume DA. Macrophages as APC and the dendritic cell myth. J Immunol (2008) 181:5829-35. doi:10.4049/jimmunol.181.9.5829

32. Steinman RM, Banchereau J. Taking dendritic cells into medicine. Nature (2007) 449:419-26. doi:10. 1038/nature06175

33. Hsieh CS, Macatonia SE, O'Garra A, Murphy KM. T cell genetic background determines default T helper phenotype development in vitro. $J$ Exp Med (1995) 181:713-21. doi:10.1084/jem.181. 2.713

34. O'Garra A, Murphy KM. Role of cytokines in determining T-lymphocyte function. Curr Opin Immunol (1994) 6:458-66. doi:10.1016/09527915(94)90128-7

35. Beutler BA. TLRs and innate immunity. Blood (2009) 113:1399-407. doi:10.1182/blood-200807-019307

36. Kawai T, Akira S. Toll-like receptors and their crosstalk with other innate receptors in infection and immunity. Immunity (2011) 34:637-50. doi:10.1016/j.immuni.2011.05.006

37. Janeway CA Jr, Medzhitov R. Innate immune recognition. Annu Rev Immunol (2002) 20:197-216. doi:10.1146/annurev.immunol. 20.083001.084359

38. Srikrishna G, Freeze HH. Endogenous damageassociated molecular pattern molecules at the crossroads of inflammation and cancer. Neoplasia (2009) 11:615-28. doi:10.1593/neo.09284

39. Matzinger P. An innate sense of danger. Ann $N$ Y Acad Sci (2002) 961:341-2. doi:10.1111/j.17496632.2002.tb03118.x

40. Mackaness GB. The immunologic basis of acquired cellular resistance. J Exp Med (1964) 120:105-20. doi:10.1084/jem.120.1.105

41. Shevach EM, Rosenthal AM. Function of macrophages in antigen recognition by guinea pig $\mathrm{T}$ lymphocytes. II. Role of the macrophage in the regulation of genetic control of the immune response. J Exp Med (1973) 138:1213-29. doi:10. 1084/jem.138.5.1213

42. Unanue ER. Antigen-presenting function of the macrophage. Annu Rev Immunol (1984) 
2:395-428. doi:10.1146/annurev.iy.02.040184. 002143

43. Dorrestein PC, Mazmanian SK, Knight R. Finding the missing links among metabolites, microbes, and the host. Immunity (2014) 40:824-32. doi:10. 1016/j.immuni.2014.05.015

44. Bae YS, Lee JH, Choi SH, Kim S, Almazan F, Witztum JL, et al. Macrophages generate reactive oxygen species in response to minimally oxidized low-density lipoprotein: toll-like receptor 4- and spleen tyrosine kinase-dependent activation of NADPH oxidase 2. Circ Res (2009) 104:210-8. doi:10.1161/CIRCRESAHA. 108.181040

45. Nathan CF. Macrophage activation: some questions. Ann Inst Pasteur Immunol (1986) 137:345-51. doi:10.1016/S0771-050X(86)80051-1

46. Hsieh CS, Macatonia SE, Tripp CS, Wolf SF, O'Garra A, Murphy KM. Development of TH1 CD4+ T cells through IL-12 produced by Listeriainduced macrophages. Science (1993) 260:547-9. doi:10.1126/science.8097338

47. Munder M, Mallo M, Eichmann K, Modolell M. Murine macrophages secrete interferon gamma upon combined stimulation with interleukin (IL)-12 and IL-18: a novel pathway of autocrine macrophage activation. J Exp Med (1998) 12:2103-8. doi:10.1084/jem.187.12.2103

48. Buhtoiarov IN, Lum H, Berke G, Paulnock DM, Sondel PM, Rakhmilevich AL. CD40 ligation activates murine macrophage via an IFN-gamma-dependent mechanism resulting in tumor cell destruction in vitro. $J$ Immunol (2005) 174:6013-22. doi:10.4049/jimmunol.174. 10.6013
49. Mosser DM, Edwards JP. Exploring the full spectrum of macrophage activation. Nat Rev Immunol (2008) 8:958-69. doi:10.1038/nri2448

50. Fleming BD, Mosser DM. Regulatory macrophages: setting the threshold for therapy. Eur J Immunol (2011) 41:2498-502. doi:10.1002/eji.201141717

51. Mantovani A, Sica A, Sozzani S, Allavena P, Vecchi A, Locati $\mathrm{M}$. The chemokine system in diverse forms of macrophage activation and polarization. Trends Immunol (2004) 25:677-86. doi:10.1016/j. it.2004.09.015

52. Stein M, Keshav S, Harris N, Gordon S. Interleukin 4 potently enhances murine macrophage mannose receptor activity: a marker of alternative immunologic macrophage activation. J Exp Med (1992) 176:287-92. doi:10.1084/jem.176.1.287

53. Martinez FO, Gordon S, Locati M, Mantovani A. Transcriptional profiling of the human monocyteto-macrophage differentiation and polarization: new molecules and patterns of gene expression. J Immunol (2006) 177:7303-11. doi:10.4049/ jimmunol.177.10.7303

54. Daley JM, Brancato SK, Thomay AA, Reichner JS, Albina JE. The phenotype of murine wound macrophages. J Leukoc Biol (2010) 87:59-67. doi: 10.1189/jlb.0409236

55. Wynn TA, Ramalingam TR. Mechanisms of fibrosis: therapeutic translation for fibrotic disease. Nat Med (2012) 18:1028-40. doi:10.1038/nm.2807

56. Bogdan C. Species differences in macrophage NO production are important. Nat Immunol (2002) 3:102. doi:10.1038/ni0202-102a

57. Fang FC, Nathan C. Man is not a mouse: reply. J Leukoc Biol (2007) 81:580. doi:10.1189/jlb. 1206715
58. Thomas A, Matilla J. Of mice and men: arginine metabolism in macrophages. Front Immunol (2014) 5:479. doi:10.3389/fimmu.2014. 00479

59. Murray PJ, Allen JE, Biswas SK, Fisher EA, Gilroy DW, Goerdt S, et al. Macrophage activation and polarization: nomenclature and experimental guidelines. Immunity (2014) 41:14-20. doi:10.1016/j.immuni.2014.06.008

60. Stout RD, Suttles J. Functional plasticity of macrophages: reversible adaptation to changing microenvironments. J Leukoc Biol (2004) 76:509-13. doi:10.1189/jlb.0504272

Conflict of Interest Statement: The authors declare that the research was conducted in the absence of any commercial or financial relationships that could be construed as a potential conflict of interest.

Received: 29 October 2014; accepted: 20 November 2014; published online: 04 December 2014.

Citation: Mills CD, Thomas AC, Lenz LL and Munder $M$ (2014) Macrophage: SHIP of Immunity. Front. Immunol. 5:620. doi: 10.3389/fimmu.2014.00620

This article was submitted to Molecular Innate Immunity, a section of the journal Frontiers in Immunology. Copyright (c) 2014 Mills, Thomas, Lenz and Munder. This is an open-access article distributed under the terms of the Creative Commons Attribution License (CC BY). The use, distribution or reproduction in other forums is permitted, provided the original author(s) or licensor are credited and that the original publication in this journal is cited, in accordance with accepted academic practice. No use, distribution or reproduction is permitted which does not comply with these terms. 